\title{
Perceptions and Metaperceptions of Negative Evaluation: Group Composition and Meta-Accuracy in a Social Relations Model
}

\author{
Alecia M. Santuzzi \\ Syracuse University
}

\begin{abstract}
This study examined whether socially stigmatized individuals (cigarette smokers) use unique person perception strategies during interaction with out-group individuals (nonsmokers), as well as implications for meta-accuracy. Undergraduate students $(N=104)$ were divided into groups of four, representing one of three compositions: all smokers, all nonsmokers, or two smokers with two nonsmokers. Participants interacted with each group member, evaluated each other, and guessed partners' evaluations of themselves (metaperceptions). Contrary to past findings, smokers' metaperceptions of out-group members were not consistent across targets. Instead, smokers' metaperceptions were influenced by the smoking status of interaction partners. Moreover, smokers interacting with nonsmokers were least accurate in their metaperceptions, compared to other dyad combinations. Connections between metaperception and meta-accuracy are discussed.
\end{abstract}

KEYWORDS meta-accuracy, metaperception, social relations model, social stigma, smoking

SMokING cigarettes is an increasingly devalued social behavior in the United States (Gibson, 1997; Kim \& Shanahan, 2003). Legislation bans smoking in public areas in many states, cities, and buildings. High taxes have been placed on the sale of cigarettes and other tobacco products. Moreover, many advertisements and products to encourage people to quit smoking are available to the public. Although the degree of negative evaluation that is attributed to smoking may depend on several factors, such as geographic region, age, and individual differences, being a smoker is generally regarded as a negative social label or stigma in contemporary US society.
Surprisingly, little empirical work has addressed the smoking stigma as it influences the social relationships among smokers and nonsmokers. Some relevant work has examined the one-sided social perceptions that nonsmokers form of smokers. For example, nonsmokers might make

\section{Author's note}

Address correspondence to Alecia M.

Santuzzi, Department of Psychology, Syracuse University, 430 Huntington Hall, Syracuse, NY 13244, USA [email: amsantuz@syr.edu or alecia2z@yahoo.com] 
more internal attributions to explain smokers' behavior relative to nonsmokers' behavior (Gibson, 1998). One study suggested that both nonsmokers and smokers believe smoking to be negative, implying that smokers had internalized the stigma of being a smoker (Goldstein, 1991). However, no empirical research to date has captured the perspectives of both nonsmokers and smokers as they interact in an interpersonal context. As a social stigma category that is often the focus of public attention in US society, smoking status is expected to play an important role in social interactions and relationships.

In order to draw inferences about relationships between stigmatized and nonstigmatized social groups, interpersonal interactions among nonstigmatized individuals, among stigmatized individuals, and between stigmatized and nonstigmatized individuals should be examined. Such a design allows for the comparison of individuals' perceptions of in-group members to those of out-group members. For example, do nonsmokers evaluate smokers differently than they evaluate other nonsmokers? Similarly, do smokers think that nonsmokers see them differently compared to how other smokers see them? Most germane to the current study, do smokers and nonsmokers accurately guess how they are evaluated by out-group members relative to in-group members? Evidence from the intergroup relations literature has highlighted some key influences that affect the sources of person perception across social categories. Such variation in person perception will likely reflect variation in the degree to which those perceptions are accurate.

\section{Types of person perception}

Two types of person perception and their relationship to each other are considered in this study. First, evaluation refers to the perception that is formed by one individual of another individual. Second metaperception, or perception of what an individual thinks another thinks about him or her, also provides important social information. Person A's metaperception of Person B's evaluation may be compared to Person B's actual evaluation of Person A in order to determine meta-accuracy, or the extent to which individuals can accurately guess what others think of them. In the present study, meta-accuracy for socially stigmatized individuals (smokers) who engage in social interaction with partners from an out-group (nonsmokers) is expected to differ from meta-accuracy during interaction with partners from an in-group.

\section{Intergroup relations and person perception}

When members of different social categories engage in social interaction, they not only form impressions of each other but also wonder what their in-group and out-group interaction partners think of them. Research by Vorauer and colleagues (Vorauer, Hunter, Main, \& Roy, 2000; Vorauer, Main, \& O'Connell, 1998) has demonstrated that members of dominant social groups expect to be negatively stereotyped by the lower-status group members. Therefore, nonstigmatized individuals might expect to be viewed negatively by stigmatized individuals during intergroup social interaction. Although this research has made an important contribution to the intergroup relations literature, one limitation is that it has captured only the perspective of nonstigmatized (high-status) individuals. Relatively little work on metaperceptions examines the dynamics of social interaction between members of nonstigmatized and stigmatized social groups (cf. Vorauer \& Kumhyr, 2001). One goal of the present study is to complement the existing work on intergroup perception and metaperception by examining both nonstigmatized and stigmatized individuals as they interact in out-group and in-group social contexts. Although the evaluations and metaperceptions of stigmatized individuals might be similar to those of nonstigmatized individuals during in-group social interaction, stigmatized individuals' metaperceptions during out-group interaction should be qualitatively different from those of nonstigmatized individuals during outgroup social interaction.

In support of the latter contention, research suggests that socially stigmatized individuals have unique experiences during social interactions (Frable, Blackstone, \& Scherbaum, 1990; Kleck \& 
Strenta, 1980; Santuzzi \& Ruscher, 2002; also see Swim \& Stangor, 1998). For instance, research has demonstrated that low-status individuals do not show the same intergroup biases, such as outgroup homogeneity and in-group favoritism, in their impressions of high-status individuals that are observed in high-status individuals' impressions of low-status individuals (Boldry \& Kashy, 1999; Major, Sciacchitano, \& Crocker, 1993; Martinot, Redersdorff, Guimond, \& Dif, 2002; also see Guinote, Judd, \& Brauer, 2002). Other research has suggested that stigmatized individuals presume that unknown interaction partners have negative attitudes toward their stigmatizing characteristics, particularly when the stigma is perceived as apparent during a social situation (Miller \& Malloy, 2003; Santuzzi \& Ruscher, 2002). These studies suggest that although stigmatized and nonstigmatized individuals may have negative expectations of what out-group members think of them, the sources behind stigmatized individuals' metaperceptions may differ from those of nonstigmatized individuals during intergroup social interaction. For example, stigmatized individuals may form negative metaperceptions of nonstigmatized others because they expect to be negatively evaluated due to their stigmas (Santuzzi \& Ruscher, 2002), whereas nonstigmatized individuals may form negative metaperceptions of stigmatized individuals because they expect a negative evaluation of the stigmatized group to be transmitted to their stigmatized interaction partners (Vorauer, 2005).

Based on past research, stigmatized group members should form metaperceptions of nonstigmatized individuals that are qualitatively distinct from those formed by nonstigmatized group members of stigmatized individuals. The present analysis further examines whether these metaperceptions reflect relatively biased or accurate representations of how out-group social interaction partners evaluate them. In other words, do stigmatized individuals accurately detect evaluations from nonstigmatized individuals? Or, do stigmatized individuals have general evaluation expectations, regardless of nonstigmatized individuals' actual evaluations of them? Assessing accuracy of metaperceptions requires information about the various sources that are driving the metaperceptions and corresponding evaluations. One method that may reveal the systematic components influencing evaluations and metaperceptions, and thus inform the interpretation of meta-accuracy, is the social relations model (Kenny, 1994).

\section{Social relations model}

The social relations model (SRM) is a method for examining perceptions and behaviors between social interaction partners that preserves the naturally occurring dyadic and reciprocal relationship effects in a social context (Kenny, 1994; also see Kenny \& LaVoie, 1984). Most of the past investigations of interpersonal and intergroup relations have focused on one or the other side of a social interaction by holding one group or group member (usually confederate) as constant while assessing the responses of the person of interest (for a review, see Hebl \& Dovidio, 2005). Such single-sided designs ignore the dynamic, interdependent processes that develop and adjust individuals' behavior during social interaction. Furthermore, such designs do not account for important influential factors that exist at the relationship (dyad or group) level, such as a shared situation. Therefore, the practice of examining one social actor who interacts with a controlled stimulus (or confederate) neglects potentially critical factors that shape interpersonal perception and compromises the validity of the conclusions that are drawn from such investigations (see Kenny \& Albright, 1987; Kenny, Bond, Mohr, \& Horn, 1996).

By taking into account the perceptions and behavior of all participants who are engaged in a particular social interaction, SRM can analyze interpersonal perceptions into both individual and dyad-level factors that may contribute to those perceptions. With multiple measures of a construct (e.g. evaluation), SRM may partition the variance of social perceptions into three major components: perceiver, target, and relationship (dyadic) variance. ${ }^{1}$ Perceiver variance represents what may be commonly interpreted as response set-how consistently individuals form impressions of their interaction partners. 
For example, if individuals tend to evaluate others in generally the same way, this would yield a large amount of variance in perceptions explained by the perceiver (i.e. perceiver variance). Target variance represents consensus or agreement in perceptions among perceivers about each target. For example, research using SRM has demonstrated that several perceivers are likely to agree on the level of extraversion in a particular target, as represented by a large target variance in interpersonal ratings of extraversion (Albright, Kenny, \& Malloy, 1988). Finally, the relationship variance represents the unique relationships between particular perceivers and particular targets. For example, a large amount of relationship variance might indicate that a particular target had an atypical relationship or interaction experience with one particular perceiver. Relationship variance is comparable to variance due to an interaction term in the more common analysis of variance (ANOVA) framework. Similar to the common ANOVA framework, relationship variance may only be interpreted as meaningful if the error (unsystematic) variance can be partialed from the variance due to the relationship. Thus, multiple measures of the same construct or the same measure across several time points must be used in order to interpret relationship variance, in addition to the perceiver and target variance components.

\section{SRM and intergroup relations}

With SRM, the question of whether interpersonal perceptions are unique (due to specific dyadic relationships) or due to a perception consistency across targets by perceivers may be addressed. That is, a perceiver may evaluate each target on the basis of idiosyncratic qualities, or evaluate targets based on a common characteristic such as group membership. Examining this type of question, Boldry and Kashy (1999) conducted an SRM study that examined out-group homogeneity and in-group bias among high-status and lowstatus individuals in a military sample. Using a combination of an asymmetric block (members of one group rate members of another group) and round-robin data structures (all individuals rate all other individuals), the authors found evidence for both in-group bias (Mullen, Brown, \& Smith, 1992) and out-group homogeneity effects (Judd \& Park, 1988), but only among the high-status group members. Low-status group members did not show such intergroup biases. Using the SRM design allows researchers to address the more refined question of whether these perception patterns are exhibited uniformly across social groups and situations. As implied by Boldry and Kashy (1999), perceptions of out-group members may vary across social groups as a function of different response set influence (e.g. stereotype use), although this work did not provide information about the degree of accuracy in these out-group judgments.

Using SRM to examine meta-accuracy of intergroup perceptions, Miller and Malloy (2003) found homosexual men to be less accurate in metaperceptions of liking by heterosexual men. However, unlike Boldry and Kashy (1999) and the present study, Miller and Malloy (2003) only examined out-group perceptions in dyads of mixed composition (e.g. one homosexual man with one heterosexual man). None of the previous studies have examined the meta-accuracy question by comparing out-group situations to exclusively in-group situations. This comparison is important because, as described earlier, interpersonal perception processes are likely to differ across in-group and out-group situations. The previous findings suggest that bearing stigmatized group membership might decrease meta-accuracy during social interaction with individuals from nonstigmatized groups. However, the relative meta-accuracy of metaperceptions of out-group members in comparison to metaperceptions of in-group members among nonstigmatized and stigmatized individuals must be examined to confirm this suggestion.

\section{SRM and meta-accuracy}

Both the definition and estimation of interpersonal accuracy have come into question. Ambiguity in the definition of the criterion for person perception accuracy is ongoing (see Funder, 1999; Kenny, 1991, 1994). Meta-accuracy refers to one type of interpersonal accuracy 
that suffers a minimal degree of ambiguity in accuracy criteria. Meta-accuracy compares Person A's evaluation of Person B to Person B's guess of what Person A's evaluation is. Thus, as long as Person B is aware of the evaluative criteria (e.g. rating items) that Person A used for the evaluation, the ambiguity in meta-accuracy criteria is conceptually minimized.

Even with relatively unambiguous conceptual criteria for meta-accuracy, the quantification of meta-accuracy remains a challenge. The most notable critique of the estimation of accuracy in person perception has been posited by Cronbach and others (e.g. Cronbach, 1955; Gage \& Cronbach, 1955; see Kenny \& Albright, 1987, for a review). The calculations that were common in the past for meta-accuracy estimation involved computing either mean differences or correlations between ratings that represented Person A's perception of Person B and Person B's guess at what Person A thinks about Person B. Both methods, particularly the use of mean differences, have been criticized for concealing important social perception information.

For example, computing a difference between means for evaluations and their corresponding metaperceptions would yield difference scores that not only neglect the magnitudes of the original ratings, but also are highly susceptible to the measurement error in the ratings (see Kenny, 1994). Difference scores do not indicate whether the accuracy or inaccuracy is a function of idiosyncratic strategies in evaluation, metaperception, or both. More importantly, differences in person perception sources may be masked by examining mean differences. In this case, Person A might guess that Person B feels very positively about him or her, and Person B indeed might evaluate Person A as positive. However, Person A might think Person B has a special interest in him or her, whereas Person B might just have a tendency to see everyone as positive. Thus, although Person A's mean metaperception seems to match Person B's mean evaluation, the sources behind those perceptions do not match. Relying on differences between means would yield misleading results.

Traditional approaches to the estimation of meta-accuracy also have employed simple correlations between evaluations and metaperceptions, using the magnitude of the correlation as the meta-accuracy index. Unlike mean difference scores, correlations have the advantage of serving as direct indexes of relatedness between sets of ratings. Whereas difference scores would provide an index of the lack of relation, correlations provide information about the degree of relation between perceptions. However, similar to difference scores, simple correlations also overlook important sources of accuracy or inaccuracy in the estimation. For example, Person B's evaluation of Person A might correlate .80 with Person A's metaperception of what Person B thinks about him or her. However, the overlap could be driven by Person A's consistency in metaperception (perhaps driven by an individual difference), Person B's general tendency to evaluate everyone that way, or a unique relationship that is shared or perceived to be shared between Person A and Person B. The information that might describe such important influences on evaluations and metaperceptions would be masked by the traditional difference score and simple correlation approaches to meta-accuracy estimation. Thus, the present study will first analyze evaluations and metaperceptions into their systematic components using the SRM, and then form correlations between these components to index meta-accuracy. This components strategy is expected to provide more precise information, relative to mean difference scores and simple correlations, about the sources in person perception and meta-accuracy during in-group and out-group social interaction.

\section{Summary}

The present study examines underlying sources in interpersonal perceptions that might change according to the situation (e.g. group composition), and how such changes might influence meta-accuracy. Based on past evidence, members of relatively stigmatized social groups should show less meta-accuracy when interacting with nonstigmatized individuals, compared to interactions with other stigmatized individuals. The social category that was highlighted in this study was smoking status (smokers v. nonsmokers). 


\section{Hypotheses}

\section{Source partitioning}

Based on previous research (Kenny \& DePaulo, 1993), both individual and shared influences were expected to drive evaluations. Specifically, evaluations of in-group and out-group members were expected to show significant perceiver and relationship variance components. As individuals in this study were only briefly acquainted, no target variance for evaluations would be expected.

Perceiver variance was expected to be the primary source of metaperceptions during in-group and out-group social interaction. However, mixed-group interactions should yield some fluctuation in the typical metaperception pattern for smokers. The source pattern in smokers' metaperceptions should be influenced by the group membership of the interaction partner. Thus, smokers' metaperceptions of nonsmokers should appear distinct in comparison to nonsmokers' metaperceptions of smokers, nonsmokers' metaperceptions of nonsmokers, and smokers' metaperceptions of smokers.

\section{Meta-accuracy}

Accuracy in metaperception should decrease among smokers when they are engaged in mixedgroup social interaction. Thus, correlations between systematic components of evaluations and metaperceptions should be lowest when smokers are forming metaperceptions of nonsmokers, relative to the other dyadic combinations. The expected decrease in meta-accuracy among smokers who interacted with nonsmokers was expected to be driven by changes in the sources of smokers' metaperceptions of nonsmokers' evaluations, rather than changes in the sources of nonsmokers' evaluations of smokers.

\section{Method}

\section{Participants}

One hundred and four students from psychology courses at Tulane University participated in a two-part study. The first part was a questionnaire session during which demographic information, including smoking status, was collected; the second part was the round-robin rating session, where both evaluations and metaperceptions of interaction partners were collected. Two groups (all smoker groups) were omitted from analysis as they failed to recall the smoking status for one or more group members. Thus, 96 participants (males $N=37$ and females $N=59$ ) comprising 24 groups (6 all nonsmokers, 6 all smokers, and 12 mixed) were included in the results that follow. ${ }^{2}$ Twice as many groups were required for the mixed-group condition as compared to the homogeneous-group conditions because each subgroup's evaluations and metaperceptions in a mixed group are analyzed as if they are separate groups (Lashley \& Kenny, 1998). With the exception of smoking status, other recorded demographic characteristics and combinations of characteristics were similar across conditions. ${ }^{3}$

\section{Materials}

Evaluations and metaperceptions were measured using a nine-item list of evaluative adjectives (see Saucier, 1994, for a similar item set). Items were selected to collectively represent general evaluation. The items included nine evaluative words: self-confident, mature, broad-minded, wise, alert, clear-headed, understanding, optimistic, and considerate. Each of these words was accompanied by the following 5-point response scale: 1 (Very little or not at all), 2 (A little), 3 (Moderately), 4 (Quite a bit), or 5 (Very much). During the round-robin interaction sessions, participants rated the interaction partner (evaluations), as well as made guesses at how partners rated them (metaperceptions) on the characteristics. Thus, each participant formed both evaluations and metaperceptions for each of the interaction partners.

\section{Part one: Smoking status}

Participants completed a brief demographics questionnaire during the first part of the study. As part of this questionnaire, various healthrelated behaviors and attitudes toward these activities were assessed. ${ }^{4}$ Of particular interest to the laboratory design, participants were asked to disclose whether they generally identify themselves as smokers (Yes or No) and their attitudes toward smoking (using a 5-point Likert response; 
-2 , Very negative to +2 , Very positive). As participants completed the survey package, each was assigned a trivial identification number to encourage the feeling of anonymity before being assigned to groups.

\section{Part two: Round-robin rating}

Based on the smoking status information that was collected during the first part, participants were assigned to groups of four for the second phase of the study. Each group represented one of three compositions: all nonsmokers, all smokers, or two smokers with two nonsmokers. As both acquaintance and prior knowledge have been suggested to improve interpersonal accuracy (see Kenny, 1994), only zero-acquaintance groups were composed prior to social interaction. An experimenter escorted each four-person group to a laboratory room and verified that none of the group members knew each other.

In the laboratory, each member of the fourperson group was given a tag with an identification letter (A, B, C, or D). Then, the experimenter used each participant's self-reported smoking status to complete a chart that indicated whether each person's letter in the four-person group indicated a smoker or a nonsmoker. Each participant received one copy of this chart. Participants were asked to circle their own letters on their respective charts to bring their attention to the smoking status of group members. The four participants were then divided into dyads and each dyad was placed in a separate room. Dyads were instructed to spend 10 minutes in a get-to-know-you conversation. Following the 10-minute interval, participants rated each other on the evaluative items. Then, each person guessed how the partner rated him or her on the same items.

After completing the discussion and ratings, dyads were switched such that each person had a new dyad partner from the four-person group. The interaction and ratings proceeded as in the previous interaction. Dyads then were switched a second time to complete the round-robin design of all possible dyad combinations within the group. Following the last dyad interaction and ratings, participants were asked to recall the smoking status of each of the group members as a check on their awareness of the group's composition.

\section{Analysis}

To assess the role of perceiver, target, and relationship variance in evaluations and metaperceptions, individual ratings for each group composition condition were analyzed into variance components using two programs-SOREMO and BLOCKO (Kenny, 1995a, 1995b). SOREMO analyzed the traditional round-robin ratings that reflected common influences of the sources among all four members of each homogeneous group. To examine the mixed groups, $B L O C K O$ performed an asymmetric block analysis that separated the smokers' out-group ratings of nonsmokers from the nonsmokers' ratings of smokers. Each variance component for evaluation and metaperception for each group composition was tested against the null hypothesis value of zero (Malloy, Agatstein, Yarlas, \& Albright, 1997). In addition, variances for nonsmokers of homogeneous groups were compared to variances for smokers of homogeneous groups, and variances for nonsmokers of mixed groups were compared to variances for smokers of mixed groups. Unfortunately, statistical comparisons of variance components among all three conditions simultaneously would not be interpretable because different variance partitioning strategies were used for homogeneous groups (roundrobin) and mixed groups (asymmetric block), each using different amounts of information. Thus, variance components and correlation results that are based on SRM analysis also are reported per group composition in a manner that allows for comparison of effect size estimates across conditions.

Meta-accuracy correlations were computed using the SRM variance partitioning for evaluations and metaperceptions within each group composition condition. For the purpose of comparison, difference scores and simple correlations between evaluations and metaperceptions were also computed using the group-level means per condition. When nonsmokers interacted only with nonsmokers and smokers interacted only 
with smokers, difference scores and correlations were computed using average ratings across all members of all groups within each condition. When interactions involved mixed groups, the difference scores and correlations between nonsmokers' metaperceptions of smokers' evaluations and smokers' evaluations of nonsmokers were computed, and differences and correlations between smokers' metaperceptions of nonsmokers' evaluations and nonsmokers' evaluations of smokers were computed. In other words, only out-group meta-accuracy estimates were formed for the mixed-group condition.

\section{Results}

\section{Smokers and nonsmokers}

As anticipated, nonsmokers $(M=-1.46, S D=0.77)$ generally reported more negative attitudes toward smoking than did smokers $(M=-0.44$, $S D=1.05),(F(1,94)=29.49, p=.00)$. This difference confirmed that smokers and nonsmokers as perceivers and targets could not be considered interchangeable. Thus, as anticipated, the two homogeneous groups were analyzed as separate conditions, and the mixed groups were analyzed as asymmetric blocks with smoking status as the subgrouping factor.

\section{Evaluation and metaperception descriptives}

All nine evaluative items were averaged to form the measurement model for a single evaluation construct. The same nine items were used to form the metaperception construct with each individual's evaluation being computed by averaging across the evaluations provided by all partners of that individual. To compute group-level means for the two homogeneous conditions (all smokers and all nonsmokers), the averages for evaluations and metaperceptions across all perceivers and targets were computed for each group, and then averaged across groups within each condition. For the mixed-group condition, averages were computed within each subgroup and included only out-group evaluations and metaperceptions. The group-level means and standard errors for evaluation and metaperception for each group composition condition are presented in Table 1.

\section{Source partitioning}

A variance component analysis using SOREMO (Kenny, 1995b) analyzed evaluations and metaperceptions into systematic and unsystematic sources of influence. The absolute variance partitioning results for the evaluation and metaperception constructs (collapsing across individual items) for each of the group composition conditions are presented in Table 2. Significance testing for the absolute variance components (see Kenny \& LaVoie, 1984; Lashley \& Bond, 1997) supported the expected pattern of results. Nonsmokers with other nonsmokers and nonsmokers with smokers showed significant perceiver components in both evaluations and metaperceptions. In addition, nonsmokers showed significant relationship components in evaluations of nonsmokers and smokers. The smokers who interacted with other smokers demonstrated strong perceiver and relationship

Table 1. Construct means by condition

\begin{tabular}{|c|c|c|c|c|}
\hline & \multirow[b]{2}{*}{ All nonsmokers } & \multirow[b]{2}{*}{ All smokers } & \multicolumn{2}{|c|}{ Mixed } \\
\hline & & & Nonsmokers & Smokers \\
\hline \multirow[t]{2}{*}{ Evaluation } & 4.14 & 3.88 & 3.77 & 3.85 \\
\hline & $(0.13)$ & $(0.11)$ & $(0.15)$ & $(0.09)$ \\
\hline \multirow[t]{2}{*}{ Metaperception } & $4.09 \mathrm{a}$ & $3.66_{\mathrm{a}}$ & 3.61 & 3.69 \\
\hline & $(0.13)$ & $(0.09)$ & $(0.10)$ & $(0.10)$ \\
\hline
\end{tabular}

Notes: Standard errors among groups within group composition condition are presented in parentheses. All nonsmoker and all smoker group means are based on six group means $(n=4)$. Each of the mixed groups are based on 12 subgroup means $(n=2)$. Common subscripts indicate that means are significantly different from each other $(p<.05)$. 
components in their evaluations and metaperceptions. However, when smokers interacted with nonsmokers, significant perceiver and relationship components emerged only for their evaluations; no source components reached statistical significance in smokers' metaperceptions of nonsmokers.

Taken together, these results demonstrate that nonsmokers' and smokers' evaluations of their interaction partners in homogeneous and mixed groups were driven by both perceiver and relationship effects. Regardless of smoking status or group composition, individuals appeared to demonstrate consistency across targets as well as develop some unique relationships with specific interaction partners that guided their evaluations of each other. Student's $t$ tests showed no statistical differences in perceiver variance or relationship variance between nonsmokers and smokers in the homogeneous groups. Similarly, paired comparisons showed no statistically significant differences in evaluation perceiver or relationship variances between nonsmokers and smokers in the mixed groups.

As expected, nonsmokers' metaperceptions of other nonsmokers, nonsmokers' metaperceptions of smokers, and smokers' metaperceptions of other smokers were influenced by perceiver effects (see Table 2). Although smokers' evaluations of nonsmokers were driven by perceiver and relationship effects, smokers' metaperceptions of nonsmokers were not driven by such sources. $t$ tests suggested that there was no statistically significant difference between homogeneous groups of nonsmokers and smokers on perceiver variance for metaperceptions. However, comparing nonsmokers to smokers in mixed groups showed that nonsmokers showed significantly more consistency in metaperceptions across targets than smokers $(t(22)=1.97, p<.05)$. Therefore, smokers, when placed in a situation where they are determining out-group attitudes toward them, appeared to be using metaperception strategies that do not reflect the typical source pattern ${ }^{5}$. Instead, the group membership of smokers' interaction partners seemed to determine whether their metaperceptions were consistent across partners. When interacting with nonsmokers, smokers did not form consistent metaperceptions.

\section{Meta-accuracy}

Difference scores and simple correlations are two traditional approaches to examining metaaccuracy. For the purposes of comparison, difference scores between metaperceptions and corresponding evaluations for each group composition condition were computed to index lack of meta-accuracy. Statistical comparisons showed significant differences neither between smokers and nonsmokers in homogeneous groups, nor between nonsmokers and smokers in mixed groups.

The simple correlations between metaperception and evaluation for each group composition condition were also computed as rough estimates

Table 2. Absolute variance partitioning for smokers and nonsmokers by condition

\begin{tabular}{|c|c|c|c|c|c|c|c|c|c|c|c|c|}
\hline & \multicolumn{6}{|c|}{ Homogeneous groups } & \multicolumn{6}{|c|}{ Mixed groups } \\
\hline & \multicolumn{3}{|c|}{ Nonsmokers } & \multicolumn{3}{|c|}{ Smokers } & \multicolumn{3}{|c|}{ Nonsmokers } & \multicolumn{3}{|c|}{ Smokers } \\
\hline & Per & Tar & Rel & Per & Tar & Rel & Per & Tar & Rel & Per & Tar & Rel \\
\hline Evaluation & $\begin{array}{c}.18^{*} \\
(.16)\end{array}$ & $\begin{array}{c}.02 \\
(.04)\end{array}$ & $\begin{array}{c}.06^{*} \\
(.06)\end{array}$ & $\begin{array}{l}.13^{*} \\
(.07)\end{array}$ & $\begin{array}{c}.07 \\
(.10)\end{array}$ & $\begin{array}{c}.06^{*} \\
(.05)\end{array}$ & $\begin{array}{c}.30 * \\
(.28)\end{array}$ & $\begin{array}{c}.08 \\
(.25)\end{array}$ & $\begin{array}{c}.11 * \\
(.19)\end{array}$ & $\begin{array}{c}.16^{*} \\
(.49)\end{array}$ & $\begin{array}{c}.03 \\
(.23)\end{array}$ & $\begin{array}{c}.10^{*} \\
(.16)\end{array}$ \\
\hline Metaperception & $\begin{array}{l}.17^{*} \\
(.10)\end{array}$ & $\begin{array}{c}.00 \\
(.02)\end{array}$ & $\begin{array}{c}.02 \\
(.03)\end{array}$ & $\begin{array}{c}.16^{*} \\
(.14)\end{array}$ & $\begin{array}{c}.01 \\
(.04)\end{array}$ & $\begin{array}{c}.04 * \\
(.02)\end{array}$ & $\begin{array}{c}.28 * \\
(.18)\end{array}$ & $\begin{array}{c}.05 \\
(.09)\end{array}$ & $\begin{array}{c}.04 \\
(.09)\end{array}$ & $\begin{array}{c}.06 \\
(.34)\end{array}$ & $\begin{array}{c}.03 \\
(.17)\end{array}$ & $\begin{array}{c}.04 \\
(.09)\end{array}$ \\
\hline
\end{tabular}

*indicates statistical significance for the one-tailed $t$ test $(p<.05$; Kenny \& LaVoie, 1984).

Notes: Perceiver (Per), target (Tar), and relationship (Rel) variances represent absolute variance estimates.

Estimates for homogeneous groups are based on four-person group ratings. Estimates for mixed groups are based on two-person subgroup ratings of out-group members. Standard deviations among groups within each condition are in parentheses. 
of meta-accuracy. Across smoker and nonsmoker in-group ratings, the correlation between metaperception and evaluation was statistically significant $(r=.90, p<.01 ; n=12)$, with smokers $(r=.95, p<.01 ; n=6)$ and nonsmokers $(r=.86$, $p=.03 ; n=6)$ showing no statistically significant difference in the degree of meta-accuracy.

When examining simple correlations in the mixed-group situation, smokers appeared to form less accurate metaperceptions of nonsmoker targets. Whereas nonsmokers seemed to show relatively high meta-accuracy when guessing how smokers had evaluated them $(r=.87, p=.02 ; n=12)$, smokers demonstrated much less accuracy in their metaperceptions of how nonsmokers had evaluated them $(r=.28$, $p=.39 ; n=12)$. Although this latter finding might seem dramatic, similar results have been demonstrated in past research on accuracy in stigmatized individuals' metaperceptions of nonstigmatized others during dyadic interaction (Miller \& Malloy, 2003).

Taken together, the traditional approaches to meta-accuracy appeared to suggest different conclusions. According to the tests on differences between metaperceptions and evaluations, neither smoking status nor group composition appeared to influence meta-accuracy. According to the simple correlation results, however, smokers seemed to show much less accuracy in their metaperceptions when they were interacting with nonsmokers (out-group members) relative to other smokers. The discrepancy in these conclusions may be resolved by using the SRM to form more precise estimates of metaaccuracy. The variance component information from the social relations analyses might suggest what would be driving the differences that were observed in the simple correlations but allow these differences to remain masked when examining the group means for each condition. In accordance with the hypothesis the unique correlation pattern in mixed-group interaction was expected to be due to an atypical pattern in smokers' metaperceptions of nonsmokers, rather than source pattern differences in the nonsmokers' evaluation of smokers. To examine this prediction, meta-accuracy correlations were computed between the systematic components of evaluations and metaperceptions for each group composition condition.

Component correlations ${ }^{6}$ between perceiver variance for metaperceptions and target variance for evaluation reflected the anticipated pattern of meta-accuracy. Both homogeneous groups showed positive perceiver-target component correlations $(r=.44$ for nonsmokers and $r=.18$ for smokers). However, the results were dramatically different for mixed groups. Nonsmokers in mixed groups were quite accurate, as measured by their perceiver-target component correlation $\left(r=1.00^{7}\right)$, whereas smokers were quite inaccurate $(r=-.83)$. To confirm this latter result, group-level perceiver-target component covariances were computed and found to be negative and statistically different from zero $(t(11)=-2.09, p<.05)$. No other dyadic compositions showed perceiver-target covariances that were statistically significant from zero at the group level. Thus, SRM component correlations confirmed that the smokers in the mixed-group situation demonstrated the least meta-accuracy. Furthermore, the meta-accuracy covariance was statistically significant and negative, suggesting that smokers might be inaccurate and biased in their metaperceptions of nonsmokers during social interaction. As nonsmokers evaluated smokers more positively, smokers perceived the evaluations as being more negative. Note that this potential bias in smokers' metaperceptions would have been masked by both the difference score and simple correlation estimates of metaaccuracy.

\section{Conclusions and discussion}

Although examination of mean differences between metaperceptions and their corresponding evaluations from others did not show any differences between nonsmokers and smokers, simple correlations demonstrated a quite dramatic pattern of meta-accuracy differences across group composition conditions. Simple metaaccuracy correlations were strong and positive for groups with only nonsmokers, only smokers, and when nonsmokers rated smokers, but a relatively small correlation emerged for smokers who rated nonsmokers. As discussed earlier, 
the conclusions based on difference scores and simple correlations may be ambiguous and run the risk of misinterpretation as they gloss over the systematic sources of interpersonal ratings. By partitioning the variance in evaluations and metaperceptions into the systematic components of those ratings, notable differences in the sources that drive interpersonal perception across social categories may be examined.

Response set differences (perceiver variance) and unique impressions (relationship variance) appeared to be strong sources in evaluation for all three group composition conditions. This would imply that the mere status of being a smoker or nonsmoker does not encourage different source patterns in interpersonal evaluations. Metaperceptions generally were driven by perceiver variance, except in mixed-group situations when ratings were made by smokers of nonsmokers; smokers in mixed groups did not demonstrate any statistically significant variance components in their metaperceptions of nonsmokers. Unlike nonsmokers, smokers' metaperceptions seemed to be sensitive to the group membership of interaction partners.

Meta-accuracy as estimated by correlations between components of the social relations analysis also seemed to be lower for smokers in the mixed group condition. Unlike metaaccuracy as estimated by difference scores or simple correlations, the social relations analysis approach allowed for the identification of the likely culprit of the inaccuracy to be the smokers' metaperceptions when interacting with nonsmokers. Furthermore, the social relations component correlations suggested that smokers' metaperceptions were not only inaccurate, but also biased, such that smokers might have misinterpreted positive information from nonsmokers as being reflections of negative attitudes.

One possible explanation for the observed bias in smokers' metaperceptions of nonsmokers that might spring to mind is that the smokers in the mixed-group situation were expecting uniform negative evaluation, which led to metaperception errors. However, based on the variance partitioning results, the observed inaccuracy is not likely due to heuristic out-group stereotypes or stereotypic expectations. The lack of a significant target component for evaluations in the mixed group situation implied that a group-specific shared stereotype was involved in neither the smokers' nor nonsmokers' evaluations of their respective out-group members. Furthermore, in the case of stereotype expectation, both the perceiver and target variance components in smokers' metaperceptions should have emerged as significant contributors during mixed-group situations. In an asymmetric analysis, metaperception perceiver variance represents variance due to one group's tendency to believe that outgroup members see them in a particular way; significant target variance in smokers' metaperceptions would imply that the smokers agreed on the metaperceptions of out-group members (i.e. shared stereotype expectation). Target variances were not significant across smoking status, group composition condition, and type of perception; thus, consensual information such as smoking-related stereotypes did not appear to be influential on intergroup perceptions in this study.

As none of the variance components in metaperceptions of smokers interacting with nonsmokers were statistically significant, factors other than perceiver, target, and relationship must be driving their perceived evaluations from others in mixed-group situations. For example, smokers might have been more focused on selfpresentation during interaction, which would not be represented as systematic variance in metaperceptions and could yield less accuracy (Albright, Forest, \& Reiseter, 2001). The results from the present study imply that smokers were less accurate when predicting what nonsmokers thought of them, but this was not a product of shared stereotype use. The specific mechanisms that drive the inaccuracy should be directly examined in future research.

\section{Caution in interpretation}

Conclusions from the meta-accuracy estimates for smokers rating nonsmokers must be interpreted with caution. The perceiver variance component for smokers' metaperceptions of nonsmokers was not statistically significant (see Table 2). Thus, the correlations that were based on this 
component might not be reliable. However, as the correlations were formed as forecast estimates without the unreliable variance (i.e. comparable to disattenuated correlations), the greater risk was that the corrected correlations would be overestimates of the true correlation. If the estimate of unreliable variance for a given systematic rating component represented only random error, then the disattenuated correlation that included this component would be the true population parameter. However, if the unreliable variance incorporates random error as well as additional sources of unaccounted variance, then omitting or 'correcting' for this variance would yield an overcorrected and overestimated correlation parameter. In the present study, the component meta-accuracy correlation for smokers interacting with nonsmokers was very large and negative. Therefore, in the case that the disattenuated correlation was inflated, the true correlation should be lower (i.e. closer to zero) than the given estimate. As the expectation was that smokers in the mixed condition would demonstrate less accuracy relative to nonsmokers and to smokers with other smokers, these potentially inflated estimates provide conservative tests of the expected relationships. The extent to which smokers' metaperceptions of nonsmokers are biased requires replication to lend credence to the conclusion.

\section{Implications for stigma and stereotyping research}

Labeling smoking as a social stigma places it in a similar category with many other characteristics that are socially stigmatized. Some commonly observed examples include non-White race, homosexuality, mental illness, HIV infection, advanced age, obesity, and chemical addictions. Similar to many other stigmatized categories, smoking is represented by a behavior (i.e. smoking a cigarette); thus, the smoker is often believed to be accountable for the condition (Jones et al., 1984). In addition, behavior-oriented stigmas are often linked to other negative characteristics, such as lack of willpower and unhealthiness (Fiske, Cuddy, Glick, \& Xu, 2002; Goldstein, 1991). However, unlike many historical stigmas, smoking may not carry with it a clear decrease in relative social power. Thus, smokers may not be deemed victims of a more privileged or dominant social category. Over time, however, smoking may become a social stigma that includes a social power differential. For instance, an individual's smoking status may influence work performance appraisals and opportunities (Gilbert, Hannan, \& Lowe, 1998), thus encouraging an unequal distribution of resources and power. The intergroup dynamics between nonsmokers and smokers should be observed over time to examine whether the social stigma of smoking becomes associated with lower social power.

In order to examine the role of social stigma during social interaction, the present study employed evaluative interpersonal judgments, rather than reports of observed behavior or trait judgments. Evaluative judgments were expected to be particularly applicable in the social interactions between individuals from different social categories. In fact, the defining feature of a social stigma is negative evaluation. The extent to which stigmatized individuals accurately detect this negative evaluation from others might allow them to better navigate their social environments. For instance, individuals may avoid experiencing stigmatization by steering away from situations where negative evaluations are especially likely. Alternatively, stigmatized individuals might use what they learn through social interaction in order to develop coping strategies to buffer negative stigma-related expectations or experiences. However, if stigmatized individuals regularly misinterpret social cues from social interaction partners, as appeared to be the case for smokers who interacted with nonsmokers in this study, the functional benefit of their strategies is likely to be minimal. Stated another way, if stigmatized individuals are overly suspicious of their interaction partners' negative evaluations of them, social functioning may be impaired and relationships with others may be less successful. On the other hand, if they ignore negative social cues, stigmatized individuals may miss costly social threats that could have been avoided or buffered if they had been recognized. Future research should address the direct implications that meta-accuracy has for stigma coping 
strategies and relationship development across social categories.

Another goal for future research should be to identify the specific mechanisms that drive the metaperceptions and meta-accuracy of individuals who are particularly motivated to control their impressions or monitor others' behavior in social situations. For example, if one social group is relatively less appealing or devalued compared to the other group, the devalued group may be motivated to defend or increase its value by showing more favorable evaluations of its own group members or less favorable evaluations of the out-group members. The valued group, on the other hand, may be motivated to maintain the status quo. Stated another way, both groups might show more negative impressions of the out-group, but they might do so for different reasons. Using this rationale, the differences in metaperceptions between nonsmokers and smokers that were observed in the present study might have been due to different motivations behind the metaperceptions. Additional research should address whether mixed-group situations invoke different person perception motivations among stigmatized compared to nonstigmatized individuals.

Although the conversation content was not monitored during this particular study, it is possible that the mixed-group situation might have cued different conversational styles and topics, relative to the homogeneous groups. Future research might examine the differences in conversation content during intergroup social interaction in order to identify the strategies that individuals use for self-presentation and stigma management. Moreover, direct observation of conversation content may provide researchers with the content of a potential stereotype of smokers in present-day society.

Traditional social psychological approaches to the examination of intergroup beliefs and attitudes have employed techniques that focus on either the nonstigmatized perpetrator or the stigmatized target's perspective during social interaction. However, such one-sided approaches do not capture the dynamic of social interaction. First, the social experiences that are examined by using one-sided observations do not reflect the reciprocal and interdependent nature of social interaction between individuals. Second, traditional simple correlation approaches to measuring meta-accuracy may be misleading as they conceal the sources of interpersonal perceptions. The social relations model addresses both of these concerns by allowing for both perceiver and target perception data. In addition, given multiple social interactions, the variance of interpersonal perceptions may be analyzed into sources to provide further information about the processes that influence interpersonal perceptions. Being able to account for sources in interpersonal perceptions has clear implications for research on social psychological phenomena, particularly those that involve intergroup relations.

The present results imply that metaperception inaccuracy is a situational and perhaps dyadic phenomenon, rather than an individual trait or skill. This suggests that both perceiver and target contribute to the accuracy or inaccuracy that occurs during person perception. The present study measured meta-accuracy with traditional difference scores and simple correlations between evaluations and metaperceptions, as well as the more refined component (perceiver-target) correlations based on SRM analysis. However, different research questions might invite different definitions of meta-accuracy. In some cases, meta-accuracy might be conceptualized as the overlap of one individual's metaperception of a specific other's evaluation (dyadic); others might see meta-accuracy as the average overlap between evaluation and metaperception across all interaction partners (generalized). Just as no one source drives interpersonal evaluations, no one definition of meta-accuracy applies to all situations. Future research should consider how differential patterns of sources in metaperception influence meta-accuracy in longterm relationships, where meta-accuracy about specific others may be more relevant than the general form of meta-accuracy addressed in the present study.

In the quest to determine the factors that drive stereotyping and prejudice, research has yielded countless results showing that individuals exhibit judgment bias when forming impressions 
of out-group members and stigmatized targets. Explanations for interpersonal judgment differences, both between and within social groups, have focused on the impact of both cognitive and affective mediating variables on differences in person perception. Some research has indicated that affect plays a strong role in the impression formation process during interpersonal interaction (e.g. Jussim, Nelson, Manis, \& Soffin, 1995). Other research has focused on the cognitive mechanisms, such as stereotype activation (e.g. Devine, 1989; Lepore \& Brown, 1997), cognitive resource depletion (e.g. Macrae, Milne, \& Bodenhausen, 1994), and motivated processing (e.g. Sinclair \& Kunda, 1999) among nonstigmatized perpetrators of prejudice. Much less research has been devoted to identifying the cognitive and affective influences on the perceptions of the stigmatized targets when interacting with nonstigmatized others (for exceptions, see Major \& Gramzow, 1999; Smart \& Wegner, 1999; Swim \& Stangor, 1998). However, the missing ingredients are the mechanisms through which these factors function to change interpersonal perceptions. Using a variance partitioning approach when examining interpersonal evaluations and metaperceptions that are formed during intergroup social interaction would give more precise information about the way in which the outcome perceptions are formed. In doing so, the definition of intergroup 'bias' may be revised to reflect the social nature of social interaction.

\section{Notes}

1. The variance partitioning approach in SRM is similar to a generalizability theory approach to interrater reliability. The main distinction is that SRM focuses on analyzing construct variance into perceiver, target, and relationship sources, whereas generalizability theory is typically employed to find target and construct sources in perceiver variance.

2. Power analyses confirmed that the number of groups for each of the three conditions yielded statistical power greater than .80 for the variance components analysis.

3. Group composition with regard to sex of participants varied within but not across conditions. No evidence suggests that sex of perceivers and targets has an impact on interpersonal accuracy in briefly acquainted groups (see Kenny, 1994); thus, these composition differences were not of great concern.

4. Self-perceptions were also measured during this phase of the study. However, the relationships between self-perceptions and the interpersonal perceptions of interest did not provide further explanation to the findings in this study. Information about the role of self-perception in this study may be requested from the author.

5. A statistical comparison between smokers in homogeneous groups and smokers in mixed groups would not be interpretable in the present study. Alternative research strategies that directly compare smokers across the two situations would provide stronger evidence for this conclusion.

6. Correlations that are based on partitioned variance components are interpreted as disattenuated correlations. Thus, component correlations are free of measurement error and represent the long-run average population estimates for meta-accuracy. Thus, statistical significance tests are not appropriate for such estimates.

7. Although a sample bivariate correlation of 1.00 is unlikely because correlations are usually influenced by measurement error to some degree. However, a component correlation that reaches 1.00 (or -1.00 ) is not uncommon. The component correlation represents the expected value for the relationship in the population after error has been removed.

\section{Acknowledgments}

This research was funded by a NIMH, Individual National Research Service Award, No. MH12915, to Alecia M. Santuzzi, in support of her predoctoral training in the Department of Psychology at Tulane University. Data collection for this work was supported by a dissertation award granted by the American Psychological Association. The continuation of this research was supported by a NIMH, National Research Service Award, No. MN14257, to the University of Illinois in support of the author as a postdoctoral trainee in the Quantitative Methods Program of the Department of Psychology, University of Illinois at Urbana-Champaign. Special thanks to 
Janet Ruscher, Ron Landis, Ed O'Neal, David Kenny, Seth Kaplan, Nyla Branscombe, and two additional reviewers for their valuable feedback on this project.

\section{References}

Albright, L., Forest, C., \& Reiseter, K. (2001). Acting, behaving, and the selfless basis of metaperception. Journal of Personality and Social Psychology, 81, 910-921.

Albright, L., Kenny, D. A., \& Malloy, T. E. (1988). Consensus in personality judgments at zero acquaintance. Journal of Personality and Social Psychology, 55, 387-395.

Boldry, J. G., \& Kashy, D. A. (1999). Intergroup perception in naturally occurring groups of differential status: A social relations perspective. Journal of Personality and Social Psychology, 77, 1200-1212.

Cronbach, L. J. (1955). Processes affecting scores on 'understanding of others' and 'assumed similarity'. Psychological Bulletin, 52, 177-193.

Devine, P. G. (1989). Stereotypes and prejudice: Their automatic and controlled components. Journal of Personality and Social Psychology, 56, $5-18$.

Fiske, S. T., Cuddy, A. J. C., Glick, P., \& Xu, J. (2002). A model of (often mixed) stereotype content: Competence and warmth respectively follow from perceived status and competition. Journal of Personality and Social Psychology, 82, 878-902.

Frable, D. E. S., Blackstone, T., \& Scherbaum, C. (1990). Marginal and mindful: Deviants in social interaction. Journal of Personality and Social Psychology, 59, 140-149.

Funder, D. C. (1999). Personality judgment: A realistic approach to person perception. San Diego, CA: Academic Press.

Gage, N. L., \& Cronbach, L. J. (1955). Conceptual and methodological problems in interpersonal perception. Psychological Review, 62, 411-422.

Gibson, B. (1997). Smoker-nonsmoker conflict: Using a social psychological framework to understand a current social controversy. Journal of Social Issues, 53, 97-112.

Gibson, B. (1998). Nonsmokers' attributions for the outcomes of smokers: Some potential consequences of the stigmatization of smokers. Journal of Applied Social Psychology, 28, 581-594.

Gilbert, G. R., Hannan, E. L., \& Lowe, K. B. (1998). Is smoking stigma clouding the objectivity of employee performance appraisal? Public Personnel Management, 27, 285-301.

Goldstein, J. (1991). The stigmatization of smokers: An empirical investigation. Journal of Drug Education, 21, 167-182.

Guinote, A., Judd, C. M., \& Brauer, M. (2002). Effects of power on perceived and objective group variability: Evidence that more powerful groups are more variable. Journal of Personality and Social Psychology, 82, 708-721.

Hebl, M. R., \& Dovidio, J. F. (2005). Promoting the 'social' in social stigmas. Personality and Social Psychology Review, 9, 156-182.

Jones, E. E., Farina, A., Hastorf, A. H., Markus, H., Miller, D. T., \& Scott, R. A. (1984). Social stigma: The psychology of marked relationships. New York: Freeman.

Judd, C. M., \& Park, B. (1988). Out-group homogeneity: Judgments of variability at the individual and group levels. Journal of Personality and Social Psychology, 54, 778-788.

Jussim, L., Nelson, T. E., Manis, M. \& Soffin, S. (1995). Prejudice, stereotypes, and labeling effects: Sources of bias in person perception. Journal of Personality and Social Psychology, 68, 228-246.

Kenny, D. A. (1991). A general model of consensus and accuracy in interpersonal perception. Psychological Review, 98, 155-163.

Kenny, D. A. (1994). Interpersonal perception: A social relations analysis. New York: Guilford.

Kenny, D. A. (1995a). BLOCKO. Unpublished computer program. University of Connecticut.

Kenny, D. A. (1995b). SOREMO. Unpublished computer program. University of Connecticut.

Kenny, D. A., \& Albright, L. (1987). Accuracy in interpersonal perception: A social relations analysis. Psychological Bulletin, 102, 390-402.

Kenny, D. A., Bond, C. F., Jr., Mohr, C. D., \& Horn, E. M. (1996). Do we know how much people like one another? Journal of Personality and Social Psychology, 71, 928-936.

Kenny, D. A., \& DePaulo, B. M. (1993). Do people know how others view them? An empirical and theoretical account. Psychological Bulletin, 114, 145-161.

Kenny, D. A., \& LaVoie, L. (1984). The social relations model. In L. Berkowitz (Ed.), Advances in experimental social psychology (Vol. 18, pp. 142-182). San Diego, CA: Academic Press.

Kim, S., \& Shanahan, J. (2003). Stigmatizing smokers: Public sentiment toward cigarette 
smoking and its relationship to smoking behaviors. Journal of Health Communication, 8, 343-367.

Kleck, R. E., \& Strenta, A. (1980). Perceptions of the impact of negatively valued physical characteristics on social interaction. Journal of Personality and Social Psychology, 39, 861-873.

Lashley, B. R., \& Bond, C. F. (1997). Significance testing for round robin data. Psychological Methods, 2, 278-291.

Lashley, B. R., \& Kenny, D.A. (1998) Power estimation in social relations analyses. Psychological Methods, 3, 328-338.

Lepore, L., \& Brown, R. (1997). Category and stereotype activation: Is prejudice inevitable? Journal of Personality and Social Psychology, 72, 275-287.

Macrae, C. N., Milne, A. B., \& Bodenhausen, G. V. (1994). Stereotypes as energy-saving devices: A peek inside the cognitive box. Journal of Personality and Social Psychology, 86, 37-47.

Major, B., \& Gramzow, R. H. (1999). Abortion as stigma: Cognitive and emotional implications of concealment. Journal of Personality and Social Psychology, 77, 735-745.

Major, B., Sciacchitano, A. M., \& Crocker, J. (1993). In-group versus out-group comparisons and selfesteem. Personality and Social Psychology Bulletin, 19, 711-721.

Malloy, T. E., Agatstein, F., Yarlas, A., \& Albright, L. (1997). Effects of communication, information overlap, and behavioral consistency on consensus in social perception. Journal of Personality and Social Psychology, 73, 270-280.

Martinot, D., Redersdorff, S., Guimond, S., \& Dif, S. (2002). Ingroup versus outgroup comparisons and self-esteem: The role of group status and ingroup identification. Personality and Social Psychology Bulletin, 28, 1586-1600.

Miller, S., \& Malloy, T. E. (2003). Interpersonal behavior, perception, and affect in status discrepant dyads: Social interaction of gay and heterosexual men. Psychology of Men $\mathcal{E}$ Masculinity, 4, 121-135.

Mullen, B., Brown, R., \& Smith, C. (1992). Ingroup bias as a function of salience, relevance, and status: An integration. European Journal of Social Psychology, 22, 103-122.

Santuzzi, A. M., \& Ruscher, J. B. (2002). Stigma salience and paranoid social cognition:
Understanding variability in metaperceptions among individuals with recently-acquired stigma. Social Cognition, 20, 171-197.

Saucier, G. (1994). Separating description and evaluation in the structure of personality attributes. Journal of Personality and Social Psychology, 66, 141-154.

Sinclair, L., \& Kunda, Z. (1999). Reactions to a Black professional: Motivated inhibition and activation of conflicting stereotypes. Journal of Personality and Social Psychology, 77, 885-904.

Smart, L., \& Wegner, D. M. (1999). Covering up what can't be seen: Concealable stigma and mental control. Journal of Personality and Social Psychology, 77, $474-486$.

Swim, J. K., \& Stangor, C. (1998). Prejudice: The target's perspective. San Diego, CA: Academic Press.

Vorauer, J. D. (2005). Miscommunications surrounding efforts to reach out across group boundaries. Personality and Social Psychology Bulletin, 31, 1653-1664.

Vorauer, J. D., Hunter, A. J., Main, K. J., \& Roy, S. A. (2000). Meta-stereotype activation: Evidence from indirect measures for specific evaluative concerns experienced by members of dominant groups in intergroup interaction. Journal of Personality and Social Psychology, 78, 690-707.

Vorauer, J. D., \& Kumhyr, S. M. (2001). Is this about you or me? Self- versus other-directed judgments and feelings in response to intergroup interaction. Personality and Social Psychology Bulletin, 27, 706-719.

Vorauer, J. D., Main, K. J., \& O’Connell, G. B. (1998). How do individuals expect to be viewed by members of lower status groups? Content and implications of meta-stereotypes. Journal of Personality and Social Psychology, 75, 917-937.

Paper received 27 June 2005; revised version accepted 10 January 2006.

\section{Biographical note}

ALECIA M. SANTUZZI is an assistant professor in the department of psychology at Syracuse University. Her research interests include intergroup relations, interpersonal perception, experiences of stigma and prejudice, and quantitative methods in social psychology. 